\title{
The Optimal Use of Triptans - Sooner is Better
}

Can. J. Neurol. Sci. 2006; 33: 3-4

The modern era of migraine pharmacotherapy began in 1926 with the introduction of ergotamine; ${ }^{1}$ however, it was another 62 years before the next significant advance in the acute treatment of migraine was realized with the introduction of sumatriptan. ${ }^{2}$ It is surprising that in 2005, some 17 years after the early reports of the use of sumatriptan for the acute treatment of severe migraine, we are only just beginning to determine the optimum way of using triptan compounds in the treatment of migraine.

Michel Ferrari and colleagues, ${ }^{3}$ in their meta-analysis of 53 trials of oral triptans in acute migraine treatment, point out that in all the trials up to 2001, patients were instructed to treat their migraine headache only when the pain was "moderate or severe on a 4 -point pain severity scale $(0=$ no pain; $1=$ mild; $2=$ moderate; $3=$ severe) and within 6-8 hours of onset". This was the standard for trial design ${ }^{4}$ and presumably was done to ensure that the headaches that were being treated really were migraine; however, it is my impression that most headache neurologists have gradually come to advise their patients, who have migraine without aura, to treat as soon as they feel the headache startdespite the lack of strong evidence to support this recommendation.

In the trials of sumatriptan therapy, the gold standard for successful headache treatment-"pain-free state within two hours of dosing"- proved to be disappointingly elusive-being obtained in only $29 \%$ of the 53 clinical trials reviewed by Ferrari et al. ${ }^{3}$ While most neurologists felt that triptans represented an improvement over ergotamine, there was still a significant group of migraineurs who were under-served by it.

In 2000, Roger Cady and colleagues ${ }^{5}$ did a retrospective analysis of data from three clinical trials of sumatriptan and found that if patients used their medication early, they were able to achieve pain-free rates at two hours of $51 \%$ (sumatriptan 50 $\mathrm{mg}$ ), $67 \%$ (sumatriptan $100 \mathrm{mg}$ ) and $28 \%$ (placebo). Furthermore, early treatment seemed to reduce the need for redosing. In that same year, Burstein and colleagues ${ }^{6}$ commented on triptan failure in migraine. They postulated that the initial throbbing phase of migraine was mediated by peripheral trigeminovascular neurons that innervate the meninges. They further postulated that later in the attack, the development of cutaneous allodynia-the perception of innocuous touch as painful-is mediated by sensitisation of central trigeminovascular neurons that receive input not only from the meninges but from the face and scalp. They found that treating migraine attacks with a triptan once allodynia (i.e. central sensitisation) had developed was ineffective at achieving a pain-free state-further supporting the idea that treating early might improve triptran efficacy. Post-hoc analysis of open-label studies of almotriptan found that when patients treated their migraine when the pain was mild, $84 \%$ were rendered pain-free at two hours compared to only $53 \%$ who waited until their pain was moderate or severe. ${ }^{7}$

Now, in this issue of the journal, Jelinski and colleagues ${ }^{8}$ report the results of a randomised, double blind, placebo controlled clinical trial of 361 patients that looks at the question of early use of triptan in the treatment of migraine. It is important to point out that the patients included in this study came from specialized headache clinics, from the practices of community neurologists and from family physicians with special interest and expertise in the management of headache. This means that the findings of this study are derived from a group of patients who probably have more severe migraine than that seen typically in the general migraine population. Nevertheless, they are probably not too different to the type of headache patient seen by most neurologists. The two-hour pain-free rates achieved by Jeliniski et $\mathrm{al}^{8}$ with early treatment with sumatriptan $100 \mathrm{mg}(50 \%)$ were significantly higher than those reported in the Ferrari metaanalysis $(29 \%) .{ }^{4}$ Interestingly, in this study, even placebo works better if taken early in the course of migraine (16\% versus $10 \%$ ) and the authors suggest that this is because, like sumatriptan, endogenous opioids - thought to be responsible for some if not all of the placebo effect-are more effective when the pain is mild. It is always nice when the results of a study confirm one's own biases. I have always used what I call "the fire analogy" with my migraine patients. I suggest to them that they should think of their migraine headache as a fire. When for example, the kitchen stove catches fire they don't pull up a chair and say to themselves, "I'll just wait and see if the fire is going to get worse" because by the time the kitchen cupboards are on fire, their little hand fire extinguisher is completely over-whelmed and they are having to call the fire department! Jelinski and colleagues have gathered good solid data that lend support for continuing to treat migraine in a way that is consistent with the fire analogy -i.e. treat the headache as soon as you are aware that it has started. What this study also shows is that despite taking sumatriptan early in the headache phase, fully one half of patients will not achieve pain-free status. Our fire extinguishers, so to speak, even when used early are imperfect. Furthermore, this was a short-term study and as the authors point out, we need to be vigilant that early treatment of mild headache does not lead to triptan over-use and eventual worsening of headache control. 
Perhaps the rate of pain-free can be further improved by taking the triptan with other medications that have been shown to be effective in the treatment of migraine-e.g. metoclopramide or a non-steroidal anti-inflammatory medication. Perhaps the use of triptans will be pushed aside, in future, by inhibitors of calcitonin gene-related peptide (CGRP), the same way that triptans have pushed aside ergotamine. Stay tuned. Undoubtedly there will be further interesting developments in this field in future.

We are told that in Egypt in $1200 \mathrm{BC}$, migraine was treated by firmly binding a clay crocodile filled with grain, using linen strips, to the head of the hapless patient. ${ }^{9}$ In the 15 th century, William Harvey recommended treating migraine with trepanation. ${ }^{9}$ The regulatory bodies of the day do not seem to have required much in the way of evidence as to the efficacy of these two procedures but the fact that they seem to have fallen out of favour is perhaps evidence enough. Who knows what the neurologist of 2500 will think looking back on the headache treatments of 2006? Perhaps many, if not all, will be quite risible; however, the collecting of good data and the making of clinical decisions based on good data, where possible, should never go out of fashion.

Paul E. Cooper London, Ontario

I should declare the following: I have received, over the years, honoraria from GlaxoSmithKlein, Merck, Novartis, Janssen-Ortho, Astra-Zeneca, Pfizer and Allergan for speaking on the treatment of headache at a variety of continuing medical education events. I have also participated on pharmaceutical industry advisory boards for eletriptan (Pfizer) and almotriptan (Janssen-Ortho). I have no other relationship with the pharmaceutical industry and do not hold shares in any of these companies.

\section{REFERENCES}

1. Maier HW. L'ergotamine inhibitteur du sympathique etudie en clinique, comme moyen d'exploration et comme agent therapeutique. Rev Neurol. 1926;33:1104-08.

2. Doenicke A, Brand J, Perrin VL. Possible benefit of GR43175, a novel 5-HT1-like receptor agonist, for the acute treatment of severe migraine. The Lancet. 1988;11:1309-11.

3. Ferrari MD, Roon KI, Lipton RB, et al. Oral tripans (Serotonin 5HT1B/1D agonists) in acute migraine treatment: a meta-analysis of 53 trials. The Lancet. 2001;358:1668-75.

4. Pilgrim AJ. Methodology of clinical trials of sumatriptan in migraine and cluster headache. Eur Neurol. 1991;31:295-9.

5. Cady RK, Sheftell F, Lipton RB, et al. Effect of early intervention with sumatriptan on migraine pain: retrospective analyses of data from three clinical trials. Clin Ther. 2000;22:1035-48.

6. Burstein R, Collins B, Jakubowski M. Defeating migraine pain with triptans: a race against the development of cutaneous allodynia. Ann Neurol. 2004;55:19-26.

7. Pascual J. Clinical benefits of early triptan therapy for migraine. Headache. 2002;42 Suppl 1:S10-7.

8. Jelinski SE, Becker WJ, Christie SN, et al. Pain free efficacy of sumatriptan in the early treatment of migraine. Can J Neuro Sci. 2006;33:73-9.

9. Edmeads J. The treatment of headache: a historical perspective. In: Gallagher RM, editor. Drug Therapy for Headache. New York: Marcel Dekker Inc; 1990. p. 108. 